\title{
Depletion of histone deacetylase 1 inhibits metastatic abilities of gastric cancer cells by regulating the miR-34a/CD44 pathway
}

\author{
LELE LIN $^{1}$, HONGPENG JIANG ${ }^{1}$, MINGKUI HUANG ${ }^{1}$, XU HOU $^{1}$, XUEPU SUN $^{1}$, XIAN JIANG ${ }^{1}$, \\ XUESONG DONG $^{1}$, XUEYING SUN ${ }^{1,2}$, BAOGUO ZHOU $^{1}$ and HAIQUAN QIAO ${ }^{1}$ \\ ${ }^{1}$ Department of General Surgery, The First Affiliated Hospital of Harbin Medical University, Harbin, Heilongjiang 150001, \\ P.R. China; ${ }^{2}$ Department of Molecular Medicine and Pathology, Faculty of Medical and Health Sciences, \\ The University of Auckland, Auckland 1005, New Zealand
}

Received January 26, 2015; Accepted April 2, 2015

DOI: $10.3892 /$ or.2015.4010

\begin{abstract}
Overexpression of histone deacetylases (HDACs) is associated with higher metastatic rates and a poor prognosis in gastric cancer. However, the underlying mechanisms involved remain unclear. The present study aimed to investigate the molecular pathways that are involved in HDAC1-mediated metastatic activities in gastric cancer cells. First we used a microRNA (miRNA or miR) microarray to screen potential miRNAs whose expression can be altered by HDAC1 depletion. Of these miRNAs, miR-34a is important as it is often inactivated in cancer cells and acts as a tumor suppressor for various types of cancer. The reverse transcription-quantitative polymerase chain reaction (RT-qPCR) results confirmed that miR-34a was upregulated by HDAC1 knockdown. Cells depleted of HDAC1 had lower abilities to migrate, invade and adhere, which were restored by a miR-34a antagomiR. Depletion of HDAC1 also resulted in impaired microfilaments and microtubules, while co-transfection of the miR-34a antagomiR attenuated these changes in the cellular cytoskeleton. The HDAC1/miR-34a axis regulated the expression and activation of CD44 and its downstream factors including Bcl-2, Ras homolog family member A (RhoA), LIM domain kinase 1 (LIMK-1) and matrix metalloproteinase (MMP)-2. The latter three proteins were responsible for the organization of tubulin and actin cytoskeleton and the formation of cellular pseudopodia. In conclusion, results of the present study indicated that HDAC1
\end{abstract}

Correspondence to: Professor Haiquan Qiao or Dr Baoguo Zhou, Department of General Surgery, The First Affiliated Hospital of Harbin Medical University, No. 23 Youzheng Road, Harbin, Heilongjiang 150001, P.R. China

E-mail: qiaohaiquan@hotmail.com

E-mail: zhoubaoguo55@163.com

Abbreviations: ERM proteins, ezrin, radixin and moesin; HDAC, histone deacetylase; LIMK, LIM domain kinase; miRNA or miR, microRNA; MMP, matrix metalloproteinase; P-LIMK, phosphorylated LIMK; RhoA, ras homolog family member A

Key words: gastric cancer, histone deacetylase 1, metastases, miR-34a, CD44 depletion inhibits the metastatic abilities of gastric cancer cells by regulating the miRNA-34a/CD44 pathway, which may be a potential target for the treatment of gastric cancer.

\section{Introduction}

Gastric cancer remains the fourth most common malignancy and the second leading cause of cancer-related mortality worldwide, although its incidence is gradually on the decrease in most parts of the world (1). The currently available chemotherapeutic agents have only limited benefits for most gastric cancer patients. Therefore, identification of novel targets and an understanding of their molecular mechanisms is crucial for the treatment of gastric cancer patients.

Histone deacetylases (HDACs) are a class of enzymes that remove the acetyl groups from core histones, as well as non-histone proteins, such as p53 (2). By modifying the status of deacetylation, HDACs induce transcriptional repression through chromatin condensation and alteration of protein activity (3). Among the 18 HDAC family members, HDAC1 accounts for more than half of cellular HDAC activity, and cannot be compensated by other HDACs (4). HDAC1 acts at all levels of gene expression including microRNA (miRNA or $\mathrm{miR}$ ) regulation (5). Overexpression of HDAC1 is significantly associated with higher lymphatic metastases and decreased 3 -year survival rates in gastric cancer patients (6). Many HDAC inhibitors have been developed, several of which have shown promise and are under investigation in clinical trials $(3,7)$. However, the underlying mechanisms for the anti-metastatic activities of HDAC inhibitors, particularly for those targeting specific HDAC1 isoform in gastric cancer, remain unclear.

miRNAs are a class of short-length (21-23 nucleotides) non-coding RNAs that regulate gene expression, and are involved in various cell functions that are essential for pathological processes in gastric cancer (8). miRNAs exert functions as tumor suppressors or oncogenes during carcinogenesis, tumor growth and metastatic dissemination (9). miR-34a is a well-known tumor suppressor that regulates the expression of Bcl-2 (10). miR-34a is widely suppressed in cancer cells, and the highly enriched miR-34a-responsive genes regulate cell proliferation, apoptosis and angiogenesis (10). Therefore, miR-34a has become a promising target for developing novel 
anticancer agents. However, it is difficult to directly manipulate miRNAs themselves, thus targeting the upstream regulators of miRNAs is a more efficient and operable approach. It has been demonstrated that the expression of miRNAs is altered by epigenetic drugs including HDAC inhibitors (11-13). In addition, the expression of miR-34a is positively regulated by p53 (14) and HDAC1 suppresses p53 activity via deacetylation (15). Therefore, HDAC1 may play a role in the metastasis of gastric cancer cells by negatively regulating miR-34a.

\section{Materials and methods}

Cell culture. Human gastric cancer SGC-7901 (metastatic carcinoma of lymph node) and MGC-803 (poorly differentiated mucinous adenocarcinoma) cells were purchased from the Type Culture Collection Cell Bank (Chinese Academy of Sciences Committee, Shanghai, China). The cells were routinely cultured in RPMI-1640 medium supplemented with $10 \%$ fetal bovine serum (FBS), $100 \mathrm{U} / \mathrm{ml}$ penicillin, and $100 \mu \mathrm{g} / \mathrm{ml}$ streptomycin at $37^{\circ} \mathrm{C}$ in a humidified atmosphere of $5 \% \mathrm{CO}_{2}$. The culture media and supplements were purchased from HyClone Co. (Logan, UT, USA).

Antibodies and related reagents. Primary antibodies (Abs) against HDAC1, Bcl-2 and glyceraldehyde-3-phosphate dehydrogenase (GAPDH) were purchased from Santa Cruz Biotechnology, Inc., Santa Cruz, CA, USA; a primary Ab against CD44 was purchased from Cell Signaling Technology, Danvers, MA, USA; and primary Abs against Ras homolog family member A (RhoA), active RhoA Ab, LIM domain kinase 1 (LIMK-1) and phosphorylated LIMK-1 (p-LIMK-1) were purchased from Abcam, Cambridge, MA, USA. A secondary horseradish peroxidase-conjugated $\mathrm{Ab}$ was obtained from the Zhongshan Golden Bridge Biotechnology Co., Ltd., Beijing, China. A primary $\mathrm{Ab}$ against $\beta$-tubulin, a biotinconjugated secondary $\mathrm{Ab}$ and a streptavidin-biotin complex (SABC)-Cy3 kit were purchased from Boster Biological Technology Co., Ltd., Wuhan, China. The fluorescein isothiocyanate (FITC)-conjugated phalloidin was purchased from Sigma, Shanghai, China.

Transfection of RNAs. RNAs used in the present study included a double-strand siRNA targeting human HDAC1 (sense, 5'-CCGGUCAUGUCCAAAGUAATT-3' and antisense, 5'-ACGUGACACGUUCGGAGAATT-3'); a double-strand siRNA targeting CD44 (sense, 5'-CUCCCAGUAUGACAC AUAUTT-3' and antisense, 5'-AUAUGUGUCAUACUGGGA GTT-3'); miR-34a mimics (sense, 5'-UGGCAGUGUCUUAGC UGGUUGU-3' and antisense, 5'-AACCAGCUAAGACACU GCCAUU-3'); a non-specific scrambled double-strand RNA (sense, 5'-UUCUCCGAACGUGUCACGUTT-3' and antisense, 5'-ACGUGACACGUUCGGAGAATT-3') serving as negative control siRNAs and miRNA mimics; a miR-34a antagomiR (5'-ACAACCAGCUAAGACACUGCCA-3'); and a scrambled control antagomiR (5'-CAGUACUUUUGUGU AGUACAA3') (GenePharma Co., Ltd., Shanghai, China). Cells were grown to $60-70 \%$ confluence, and incubated with RNAs at a final concentration of $0.1 \mu \mathrm{M}$ using Lipofectamine ${ }^{\mathrm{TM}} 2000$ (Invitrogen, Beijing, China) in a serum-free medium for $48 \mathrm{~h}$ and then subjected to assays.
miRNA microarray analysis. Total RNA was extracted using a TRIzol (Invitrogen) and RNeasy Mini kit (Qiagen, Hilden, Germany), and RNA concentrations quantified with the NanoDrop NP-1000. The samples were labeled using the miRCURY ${ }^{\mathrm{TM}} \mathrm{Hy}^{\mathrm{TM}} / \mathrm{Hy}^{\mathrm{TM}}$ Power labeling kit and hybridized on the miRCURY ${ }^{\text {тм }}$ LNA Array (v.18.0) (both from Exiqon, Vedbaek, Denmark). Slides were washed using a wash buffer (Exiqon), dried and scanned on an Axon GenePix 4000B microarray scanner (Molecular Devices, Downingtown, PA, USA). GenePix pro V6.0 (Molecular Devices) was used to measure the raw intensity. After background subtraction and normalization, the results were subjected to unsupervised hierarchical clustering (Cluster 3.0).

Reverse transcription-quantitative PCR (RT-qPCR). The RT-qPCR of mature miRNAs was performed using TaqMan miRNA assays. Total RNA was harvested as above, and complementary DNA was synthesized with a GeneAmp PCR System 9700 (Applied Biosystems, Foster City, CA, USA) using an MMLV reverse transcriptase (Epicentre, Madison, WI, USA). RT-qPCR was performed by an ABI PRISM 7900 system (Applied Biosystems). Experiments were performed in triplicate, and data were presented as fold changes calculated by the $2^{-\Delta \Delta \mathrm{Ct}}$ method. The levels of miRNAs were normalized against U6 controls. The two reverse transcription primers: 5'-GTCGTATCCAGTGCGTGTCGTGGAGTCGGCAATTG CACTGGATACGACACAACCAG-3', and 5'-CGCTTCACG AATTTGCGTGTCAT-3' were designed for miR-34a and U6, respectively. Two pairs of RT-qPCR primers were designed for miR-34a-5p (forward, 5'-AGGGGGTGGCAGTGTCTTAG-3' and reverse, 5'-GTGCGTGTCGTGGAGTCG-3'); and U6 (forward, 5'-GCTTCGGCAGCACATATACTAAAAT-3' and reverse, 5'-CGCTTCACGAATTTGCGTGTCAT-3'). The primers were obtained from Invitrogen (Shanghai, China).

Immunoblotting assay. An immunoblotting assay was performed as previously described $(16,17)$. Briefly, the cells were lysed and protein concentrations were quantified. Cell lysates were separated by SDS-PAGE and transferred onto polyvinylidene difluoride (PVDF) membranes (Millipore, Bedford, MA, USA). After blocking with 5\% non-fat milk, blots were probed overnight at $4{ }^{\circ} \mathrm{C}$ with primary Abs (dilutions: anti-HDAC1, 1:200; anti-CD44, 1:200; anti-Bcl-2, 1:200; anti-RhoA, 1:1,000; anti-LIMK-1, 1:1,000; anti-P-LIMK-1, and 1:1,000; anti-GAPDH, 1:2,000), and then incubated with secondary Abs (1:1,000 dilution). The signal was detected by BeyoECL plus reagent (Beyotime Institute of Biotechnology, Jiangsu, China) and visualized using a ChemiDoc ${ }^{\mathrm{TM}} \mathrm{XRS}+$ System (Bio-Rad Laboratories, Inc., Hercules, CA, USA). The density of each band was measured using Image $\mathrm{Lab}^{\mathrm{TM}}$ software (Bio-Rad Laboratories, Inc.).

Cell migration and invasion assays. Cell migration and invasion assays were performed as previously described (18). For the migration assays, $2.5 \times 10^{4}$ cells suspended in $500 \mu 1$ serum-free RPMI-1640 medium were seeded in BD Falcon cell culture inserts ( $8-\mu \mathrm{m}$ pore; BD Biosciences, San Jose, CA, USA). Medium supplemented (750 $\mu \mathrm{l})$ with $10 \%$ FBS was added into the wells of the BD Falcon TC companion plate (BD Biosciences) as the chemoattractant. After incubation for 
Table I. Alteration of miRNA expression induced by HDAC1 siRNA in gastric cancer cells.

\begin{tabular}{|c|c|c|c|c|c|c|c|}
\hline $\begin{array}{l}\text { Upregulated } \\
\text { miRNAs }\end{array}$ & $\begin{array}{l}\text { Control } \\
\text { siRNA }\end{array}$ & $\begin{array}{l}\text { HDAC1 } \\
\text { siRNA }\end{array}$ & Fold-change & $\begin{array}{c}\text { Downregulated } \\
\text { miRNAs }\end{array}$ & $\begin{array}{l}\text { Control } \\
\text { siRNA }\end{array}$ & $\begin{array}{l}\text { HDAC1 } \\
\text { siRNA }\end{array}$ & Fold-change \\
\hline hsa-miR-20a-5p & 0.18565 & 4.47384 & 24.097710 & hsa-miR-342-5p & 0.46203 & 0.01744 & 0.037751 \\
\hline hsa-let-7b-3p & 0.01477 & 0.32267 & 21.849670 & hsa-miR-449c-3p & 0.26160 & 0.01163 & 0.044449 \\
\hline hsa-miR-196b-3p & 0.27637 & 2.22093 & 8.036038 & hsa-miR-222-5p & 0.37764 & 0.02035 & 0.053885 \\
\hline hsa-miR-10b-5p & 0.08228 & 0.65988 & 8.020125 & hsa-miR-182-5p & 0.18565 & 0.01163 & 0.062632 \\
\hline hsa-miR-29a-3p & 0.07384 & 0.38953 & 5.275415 & hsa-miR-4319 & 0.21941 & 0.01453 & 0.066246 \\
\hline hsa-miR-17-5p & 0.09283 & 0.4564 & 4.916623 & hsa-miR-675-5p & 0.93882 & 0.07558 & 0.080507 \\
\hline hsa-miR-92b-3p & 0.26793 & 1.29651 & 4.838949 & hsa-miR-320c & 0.50211 & 0.04070 & 0.081053 \\
\hline hsa-miR-34a-5p & 0.07384 & 0.31686 & 4.291196 & hsa-miR-424-5p & 1.52954 & 0.12500 & 0.081724 \\
\hline hsa-miR-196a-3p & 0.40084 & 1.57849 & 3.937913 & hsa-miR-450a-5p & 0.34599 & 0.02907 & 0.084019 \\
\hline hsa-miR-10a-5p & 0.10759 & 0.40988 & 3.809508 & hsa-miR-221-3p & 0.66667 & 0.06105 & 0.091570 \\
\hline hsa-miR-24-1-5p & 1.12236 & 4.18605 & 3.729673 & hsa-miR-26a-2-3p & 0.28059 & 0.03198 & 0.113962 \\
\hline hsa-miR-1246 & 17.35230 & 64.6047 & 3.723113 & hsa-miR-652-3p & 0.22785 & 0.03198 & 0.140342 \\
\hline hsa-miR-382-5p & 0.06329 & 0.23547 & 3.720349 & hsa-miR-424-3p & 1.09494 & 0.15698 & 0.143366 \\
\hline hsa-miR-30b-5p & 0.14768 & 0.53198 & 3.602243 & hsa-miR-431-5p & 0.52321 & 0.08140 & 0.155570 \\
\hline hsa-miR-320a & 0.52743 & 1.87209 & 3.549488 & hsa-miR-181c-3p & 0.22152 & 0.03488 & 0.157475 \\
\hline hsa-miR-133b & 0.41772 & 1.44477 & 3.458686 & hsa-miR-1296 & 0.36076 & 0.06105 & 0.169217 \\
\hline hsa-miR-340-5p & 0.31013 & 1.06105 & 3.421334 & hsa-miR-204-5p & 0.25105 & 0.04360 & 0.173686 \\
\hline hsa-miR-193a-3p & 0.58861 & 1.99419 & 3.387972 & hsa-miR-7-5p & 0.41983 & 0.07849 & 0.186952 \\
\hline hsa-miR-518b & 0.09916 & 0.32558 & 3.283523 & hsa-miR-499a-3p & 0.13713 & 0.02616 & 0.190787 \\
\hline hsa-miR-361-3p & 0.39662 & 1.28488 & 3.239547 & hsa-miR-486-3p & 0.44937 & 0.09302 & 0.207009 \\
\hline hsa-miR-18b-3p & 0.98734 & 3.15116 & 3.191562 & hsa-miR-493-5p & 0.22363 & 0.06105 & 0.272982 \\
\hline \multirow[t]{4}{*}{ hsa-miR-199a-5p } & 0.29325 & 0.90698 & 3.092856 & hsa-miR-659-3p & 0.79325 & 0.23547 & 0.296836 \\
\hline & & & & hsa-miR-564 & 3.03797 & 0.92151 & 0.303331 \\
\hline & & & & hsa-miR-29b-3p & 0.20464 & 0.06395 & 0.312515 \\
\hline & & & & hsa-miR-96-5p & 0.14557 & 0.04942 & 0.339484 \\
\hline
\end{tabular}

SGC-7901 cells were transfected with control or HDAC1 siRNAs, harvested $48 \mathrm{~h}$ later and subjected to a miRNA microarray. The expression level of each miRNA was measured and the fold-change was calculated. The 47 differentially expressed miRNAs that matched the 4-fold threshold are listed only. HDAC1, histone deacetylase 1.

$24 \mathrm{~h}$, the non-migrating cells were removed from the upper surface of the membrane by cotton swabs. The cells on the lower surface were fixed in $4 \%$ paraformaldehyde, stained with Giemsa reagent (Jiancheng Biotechnology, Nanjing, China) and air dried. The cells were then counted at a magnification of x100 in five random fields, using an IX71 inverted phase contrast fluorescence microscope (Olympus, Tokyo, Japan) and photographed with a digital camera. For the invasion assays, the method was similar to that of the migration assays. The cells were placed into the BD BioCoat Matrigel invasion chambers (8- $\mu \mathrm{m}$ pore; BD Biosciences), which were coated with the Matrigel matrix and rehydrated for $2 \mathrm{~h}$ with pre-warmed culture medium at $37^{\circ} \mathrm{C}$, in a humidified atmosphere of $5 \% \mathrm{CO}_{2}$. After incubation for $48 \mathrm{~h}$, the non-invading cells were removed. The cells attached to the lower surface were then fixed, stained, air dried, counted and photographed.

Cell adhesion assay. Plates (96-well) were coated with fibronectin $(80 \mu \mathrm{g} / \mathrm{ml}$; Sigma, St. Louis, MO, USA) overnight at $4^{\circ} \mathrm{C}$, and blocked with $2.0 \%$ bovine serum albumin at $37^{\circ} \mathrm{C}$ for $1 \mathrm{~h}$ in a humidified incubator. The cells were seeded at a concentration of $1 \times 10^{4}$ cells/well and allowed to adhere for
$1 \mathrm{~h}$. The non-adherent cells were then washed away with phosphate-buffered solution and the adherent cells were subjected to CCK-8 assays to read optical density (OD) values using a CCK-8 kit (Dojindo Molecular Technologies, Beijing, China).

Immunofluorescence assay. The cells were fixed with $4 \%$ paraformaldehyde and then permeabilized in $0.1 \%$ Triton $\mathrm{X}-100$. For $\beta$-tubulin staining, the cells were blocked in $10 \%$ normal goat serum, incubated with the anti- $\beta$-tubulin $\mathrm{Ab}$ for $90 \mathrm{~min}$, followed by incubation with the biotin-conjugated secondary $\mathrm{Ab}$ for $30 \mathrm{~min}$, and then stained with the SABCCy 3 for $30 \mathrm{~min}$. For F-actin staining, the cells were stained with FITC-conjugated phalloidin for $40 \mathrm{~min}$. The cells were then counterstained with 4',6-diamidino-2-phenylindole (DAPI) for $15 \mathrm{~min}$, and images were captured with an IX71 inverted phase contrast fluorescence microscope (Olympus).

Gelatin zymography. A gelatin zymography assay was performed as previously described (18). Cells $\left(30.0 \times 10^{4}\right)$ were seeded in 6-well plates and starved with serum-free medium for $24 \mathrm{~h}$. The conditioned medium was collected and ultracentrifuged. The respective centrifuged medium $(40 \mu \mathrm{l})$ 
A

HDAC1

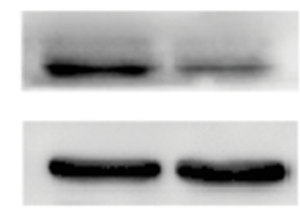

GAPDH

$\mathrm{C}$

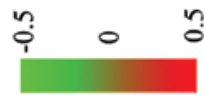

B

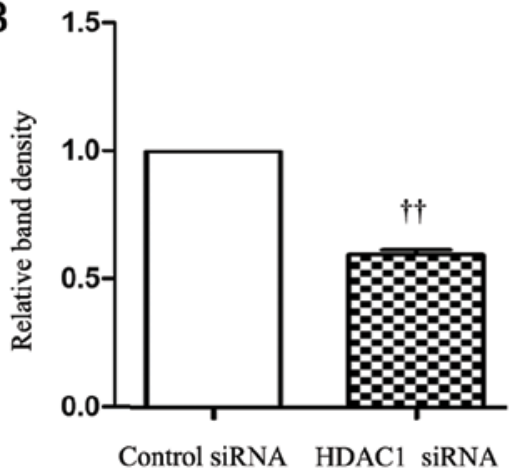

D

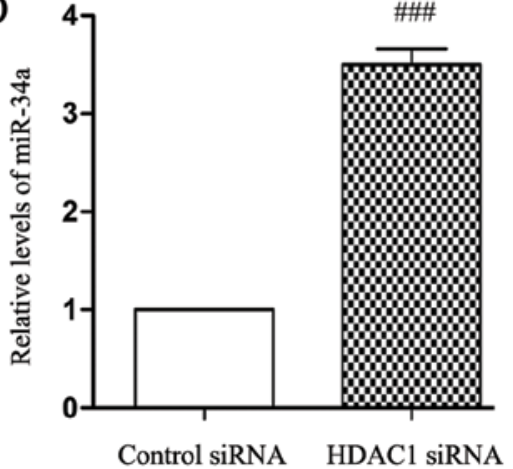

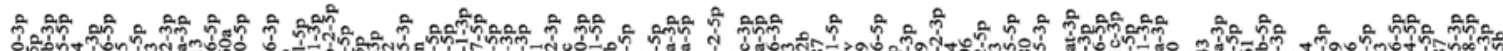

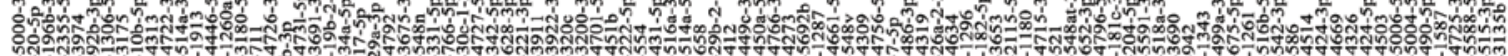

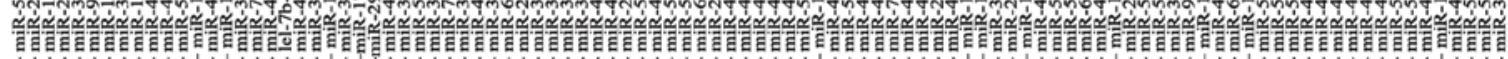

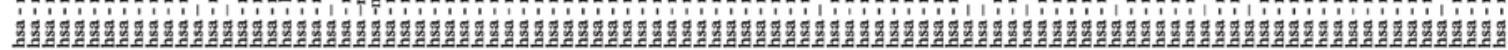

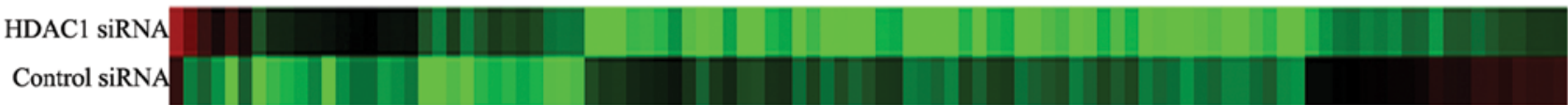

Figure 1. Knockdown of HDAC1 upregulates the expression of miR-34a. SGC-7901 cells were transfected with control or HDAC1 siRNAs, and harvested $48 \mathrm{~h}$ later. (A) Cell lysates were immunoblotted to detect the expression of HDAC1. (B) The density of each band was measured and normalized to GAPDH (B). The relative band density from control cells was defined as 1. (C) Total RNA was extracted and the expression signature of miRNAs in SGC-7901 cells transfected with HDAC1 or control siRNAs was analyzed by a miRNA microarray. The heat map presents the expression profiles of 101 differentially expressed miRNAs matching the 4-fold threshold. Each column shows a miRNA and each row a sample. The relative ratios of expression are indicated by color: Red, a relatively higher expression level, and green, a relatively lower expression level. (D) Total RNA was extracted and the expression level of miR-34a was determined by qRT-PCR. The level of mRNA from control cells was defined as $1 .{ }^{\dagger \dagger} \mathrm{P}<0.01$, indicates a significant reduction, and ${ }^{\# \# \#} \mathrm{P}<0.001$, a significant increase versus the controls. HDAC1, histone deacetylase 1.

was separated by $10 \%$ SDS-PAGE gels containing $2 \mathrm{mg} / \mathrm{ml}$ gelatin (Sigma) on ice under non-reducing conditions. After the electrophoresis, the gels were incubated in the zymogram renaturing buffer (2.5\% Triton X-100 in deionized water) twice with gentle agitation for $40 \mathrm{~min}$ at room temperature. The zymogram renaturing buffer was decanted and replaced with zymogram-developing buffer (50 mM Tris- $\mathrm{HCl}, 5 \mathrm{mM}$ $\mathrm{CaCl}_{2}, 0.02 \%$ Brij-35, 0.2 M NaCl, pH 7.6). The gels were equilibrated for $30 \mathrm{~min}$ at room temperature with gentle agitation, and incubated with a fresh zymogram-developing buffer at $37^{\circ} \mathrm{C}$ for $42 \mathrm{~h}$. Subsequently, the gels were stained with $0.1 \%(w / v)$ Coomassie blue R-250 for $30 \mathrm{~min}$ at room temperature, followed by destaining with an appropriate Coomassie blue R-250 destaining solution [methanol, acetic acid, water (30:10:60)]. Clear bands against the blue background represented areas of gelatinolysis. Those bands were photographed using a ChemiDoc ${ }^{\mathrm{TM}}$ XRS+ System (Bio-Rad Laboratories, Inc.).

RhoA-GTP pull-down assay. Active RhoA was pulled down from whole cell lysates using a RhoA Activation Assay kit (NewEast Biosciences, Malvern, PA, USA). Briefly, the cells were grown to $80-90 \%$ confluence, lysed in the presence of protease inhibitors, and centrifuged. The supernatant was incubated with anti-active RhoA $\mathrm{Ab}$ (1:1,000 dilution) and protein $\mathrm{A} / \mathrm{G}$ Agarose bead slurry at $4^{\circ} \mathrm{C}$ for $1 \mathrm{~h}$. The beads were washed and resuspended in SDS-PAGE sample buffer, and GTP-bound RhoA was immunoblotted.
Statistical analysis. Experiments were performed in triplicate and repeated at least three times. Data are presented as mean values \pm standard deviation. Comparisons were made using one-way analysis of variance followed by Tukey's post-hoc test with SPSS statistical software (version 21.0). $\mathrm{P}<0.05$ was considered to indicate a statistically significant result.

\section{Results}

Depletion of HDACl results in the upregulation of miR-34a in gastric cancer cells. First, we used a specific siRNA to knock down the expression of HDAC1 in SGC-7901 cells. As shown in Fig. 1A and B, the expression of HDAC1 was significantly downregulated $48 \mathrm{~h}$ after HDAC1 siRNA transfection, while the control siRNA had no effect on the expression of HDAC1. We then examined the global miRNA expression in cells transfected with control and HDAC1 siRNAs using a miRCURY'T LNA Array (Fig. 1C and Table I). The miRNA array data supported our hypothesis that the expression of miR-34a was significantly upregulated by knockdown of HDAC1. Consistently, the RT-qPCR analysis confirmed that HDAC1 siRNA transfection significantly increased the cellular expression level of miR-34a by $\sim 4$-fold compared to the control siRNA (Fig. 1D).

Knockdown of HDACl inhibits the abilities of gastric cancer cells to migrate, invade and adhere by regulating miR-34a. It has been reported that a high expression of HDAC1 is 
A SGC-7901

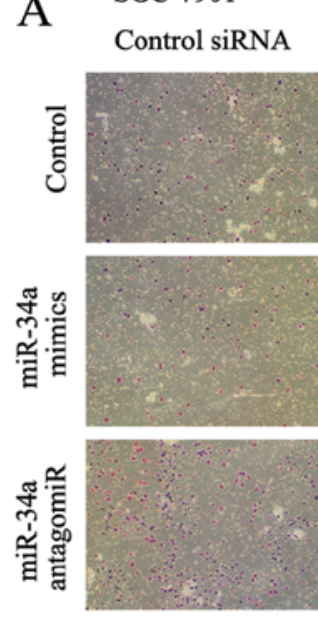

C
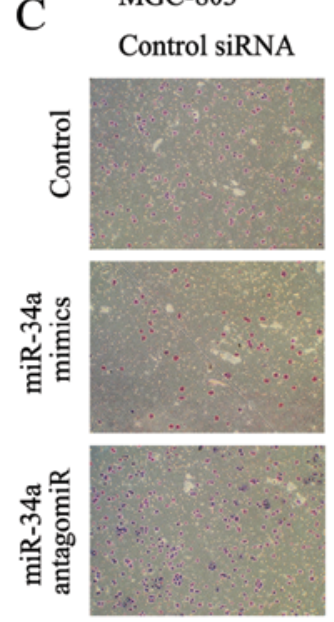

G

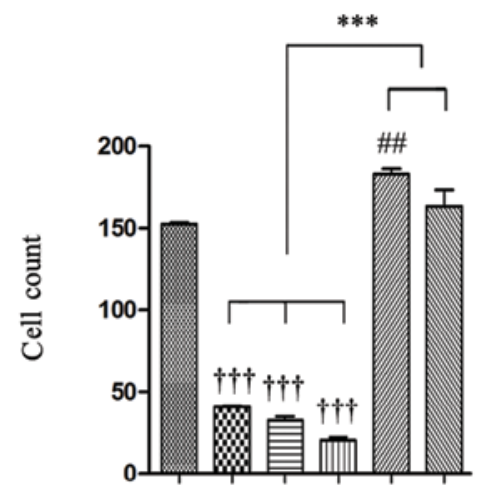

HDAC1 siRNA - + - + - +

miR-34a mimics - - + + - -

miR-34a antagomiR - $\quad-\quad-\quad+\quad+$
B

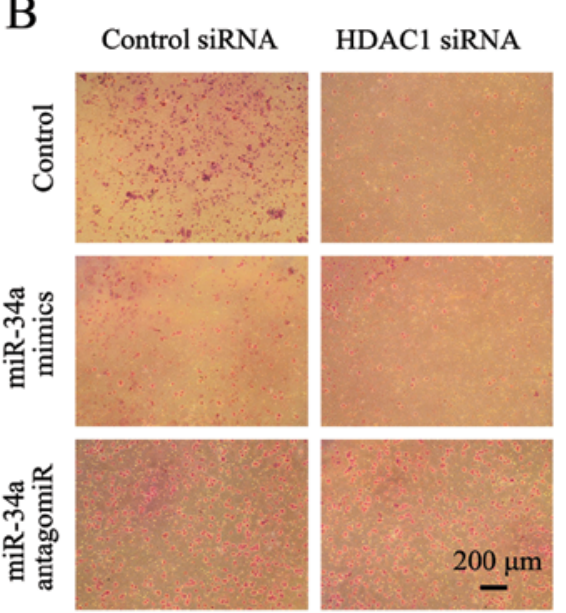

D
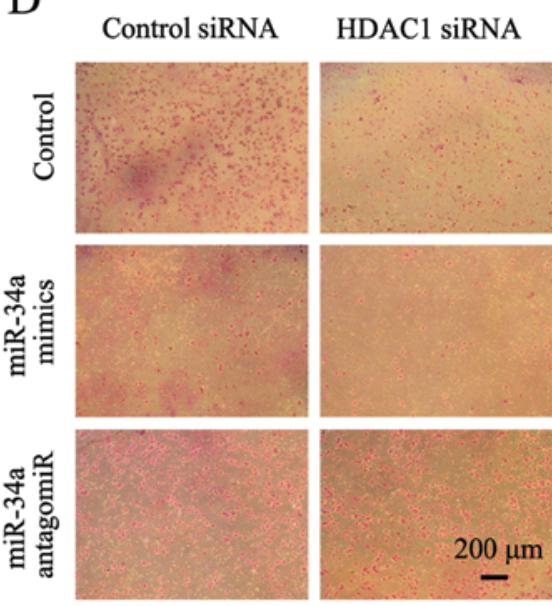

$\mathrm{H}$

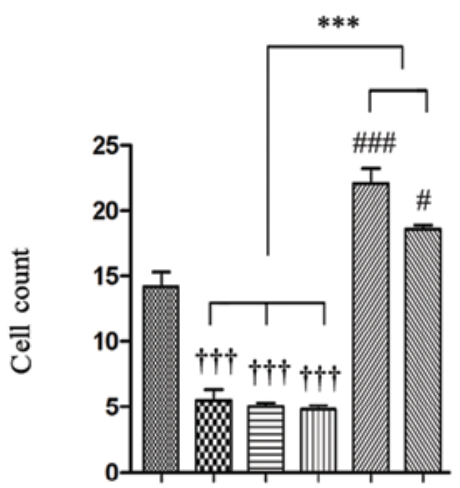

HDAC1 siRNA - + - + - +

miR-34a mimics - - + + - -

miR-34a antagomiR - $\quad-\quad+\quad+\quad+$
E

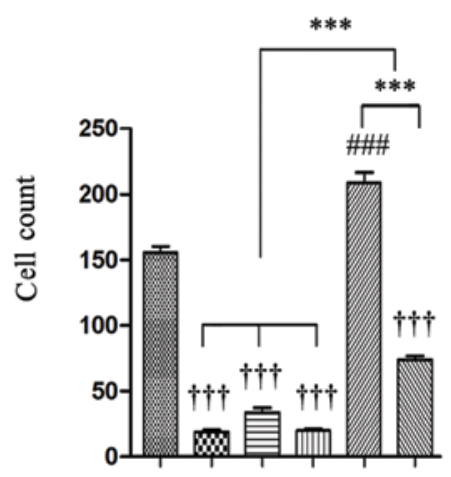

HDAC1 siRNA - + - + - +

miR-34a mimics - - + + - -

miR-34a antagomiR - - - - ++

F

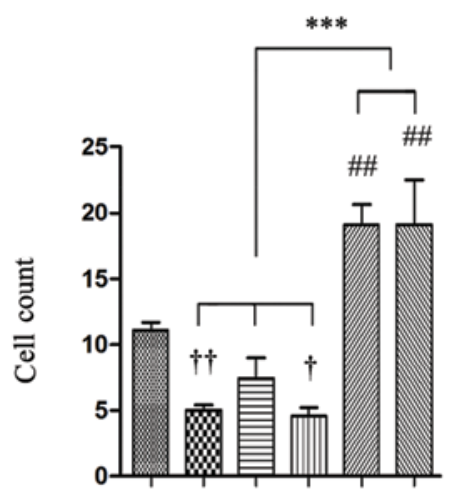

HDAC1 siRNA - + - + - +

miR-34a mimics - - + + - -

miR-34a antagomiR - - - - ++
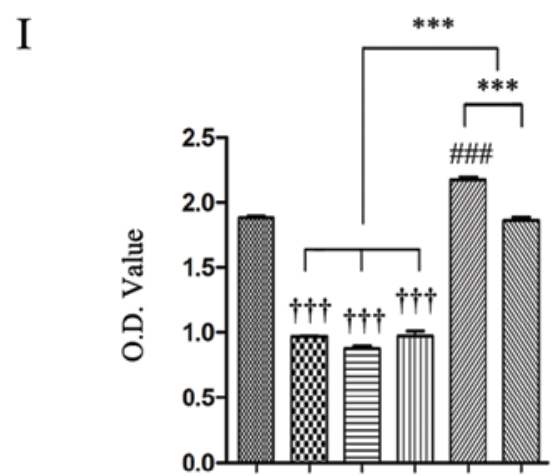

HDAC1 siRNA - + - + - +

miR-34a mimics - - + + - miR-34a antagomiR - - - - + +

Figure 2. Knockdown of HDAC1 inhibits the abilities of cells to migrate, invade and adhere via miR-34a. SGC-7901 and MGC-803 cells were transfected with control or HDAC1 siRNAs in the presence of control or miR-34a mimics or miR-34a antagomiR for $48 \mathrm{~h}$, and then subjected to migration, invasion and adhesion assays as described in Materials and methods. (A-D) Representative images were captured from the migrated (A and C) and invaded (B and D) cells (magnification, x100). (E-H) The number of migrated (E and G) and invaded ( $\mathrm{F}$ and $\mathrm{H}$ ) cells were counted. (I) The adhered cells were subjected to a CCK-8 assay to measure OD (optical density) values. ${ }^{\dagger} \mathrm{P}<0.05,{ }^{\dagger} \mathrm{P}<0.01$ and ${ }^{\dagger \dagger} \mathrm{P}<0.001$ indicate a significant reduction, and ${ }^{\#} \mathrm{P}<0.05,{ }^{\# \#} \mathrm{P}<0.01$ and ${ }^{\# \# \#} \mathrm{P}<0.001$, a significant increase, from cells transfected with control siRNA and control antagomiR. ${ }^{* * *} \mathrm{P}<0.001$, a significant difference. HDAC1, histone deacetylase 1 .

positively correlated with metastasis in gastric cancer (6), thus we examined whether HDAC1 depletion affects the ability of SGC-7901 and MGC-803 cells to migrate, invade and adhere. Transfection of HDAC1 siRNA led to markedly reduced number of SGC-7901 cells (Fig. 2A and B) and MGC-803 cells (Fig. 2C and D) that had migrated and invaded through the Transwell membranes. Quantitative analysis confirmed that the numbers of migrated and invaded SGC-7901 cells 


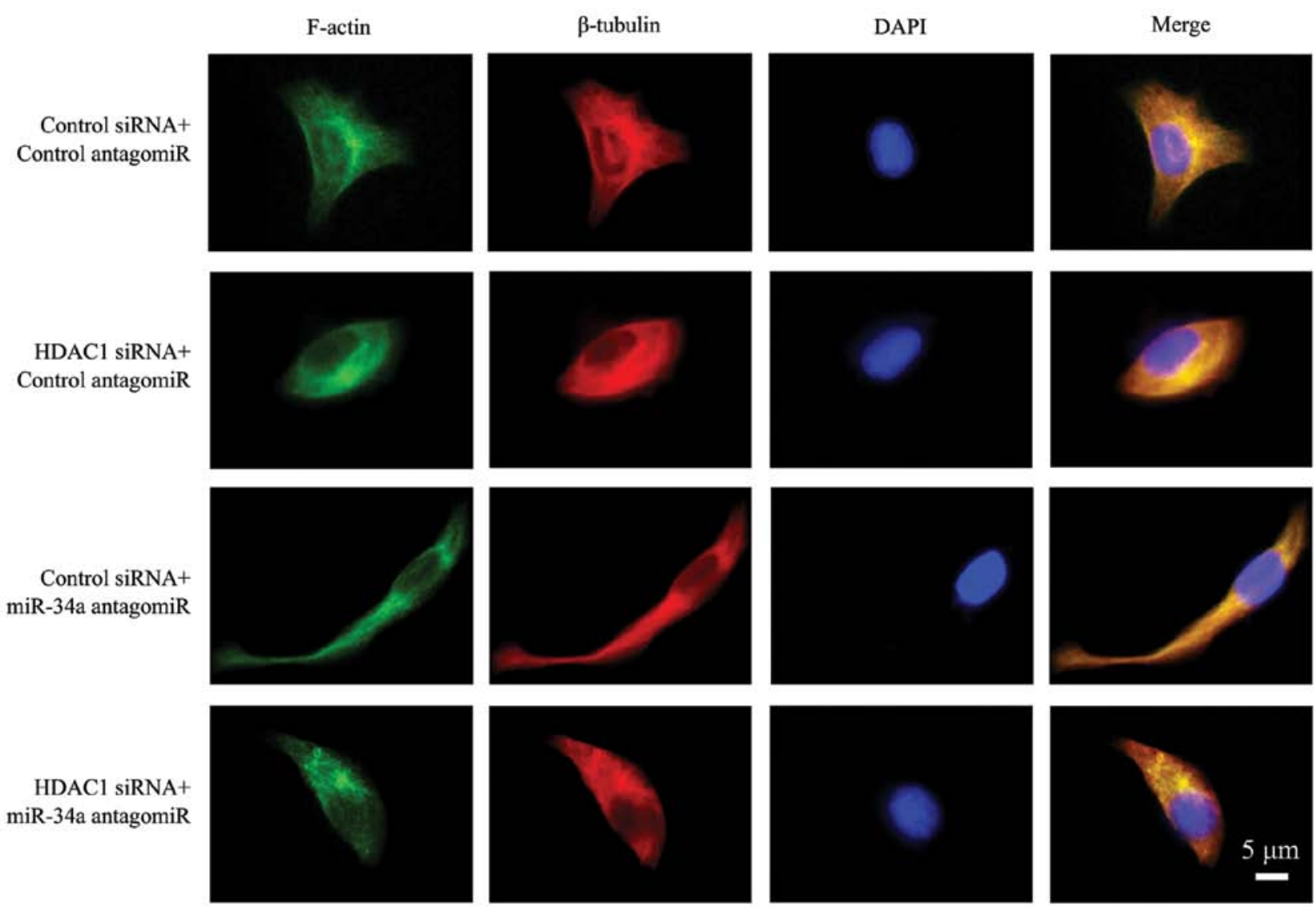

Figure 3. The effects of HDAC1/miR-34a on cellular cytoskeleton. The cells in Fig. 2 were subjected to immunofluorescence assay. F-actin was immunostained by phalloidin for the presence of microfilaments (green); $\beta$-tubulin was detected by an anti- $\beta$-tubulin antibody for the presence of microtubules (red); and cell nuclei were counterstained by DAPI (blue). The three images (magnification, $\mathrm{x} 400$ ) from the same cells were merged to produce virtual color (red, green and blue) images. HDAC1, histone deacetylase 1.

were highly significantly lower by 86.7 and $54.5 \%$, respectively, in HDAC1 siRNA-transfected cells, compared with controls (Fig. 2E and F), and the number of those MGC-803 cells was significantly lower by 73.4 and $61.1 \%$ (Fig. 2G and H). In miR-34a mimics-transfected cells, the numbers of migrated and invaded cells were markedly decreased compared with the controls, while co-transfection with HDAC1 siRNA and miR-34a mimics further decreased those numbers (Fig. 2E-H). By contrast, transfection of a miR-34a antagomiR significantly increased the numbers of migrated and invaded cells compared with the controls. In addition, when cells were co-transfected with HDAC1 siRNA and miR-34a antagomiR, the number of migrated cells was significantly lower than cells transfected with miR-34a antagomiR alone, and significantly higher than those transfected with HDAC1 siRNA alone (Fig. 2E-H). HDAC1 siRNA or miR-34a mimics significantly reduced, while miR-34a antagomiR significantly increased, the number of adhered cells as expressed by OD values, compared with the controls (Fig. 2I). Co-transfection with HDAC1 siRNA and miR-34a antagomiR resulted in a significantly lower number of adhered cells than miR-34 antagomiR alone, and a significantly higher number of adhered cells than HDAC1 siRNA alone (Fig. 2I).

HDACl and miR-34a axis regulates cellular cytoskeleton. The above cells were immunostained with phalloidin for the presence of microfilaments, an anti- $\beta$-tubulin $\mathrm{Ab}$ for the presence of microtubules, and DAPI for the nuclei. As shown in Fig. 3, the cells transfected with control siRNA and antagomiR presented clear and intact microfilaments, and intact microtubules with well-formed dendrites. In cells depleted of HDAC1, microfilaments and microtubules were impaired and blurred, leading to a rounder cell appearance without obvious pseudopodia. The cells transfected with miR-34a antagomiR had a more stretched arrangement of microfilaments and microtubules, with obvious spread-out and long dendrites. Co-transfection with miR-34a antagomiR partially restored the impaired microfilaments and microtubules in cells transfected with HDAC1 siRNA. However, the cells still showed a poorly organized cytoskeleton and weakened dendrites (Fig. 3).

HDACl/miR-34a regulates the expression of CD44 in gastric cancer cells. CD44 is a cell-surface adhesion glycoprotein involved in cell adhesion, homing, migration and apoptosis resistance and the development of anti-CD44 tumor-specific agents has become a realistic therapeutic approach (19). In the present study, we demonstrated that the transfection of miR-34a mimics significantly downregulated, while miR-34a antagomiR upregulated, the expression of CD44 in gastric cancer cells, compared with control mimics (Fig. 4A and B). Transfection of HDAC1 siRNA significantly reduced the expression of CD44 and Bcl-2 (Fig. 4C and D), with the latter being a well-studied 
D

A

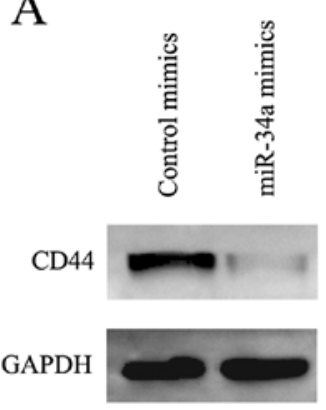

C
B

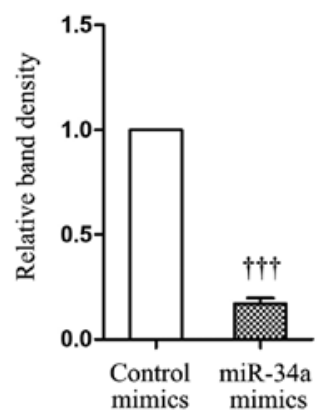

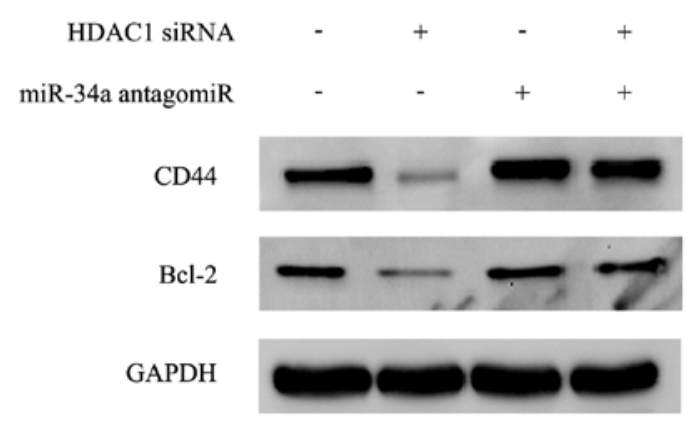

CD44

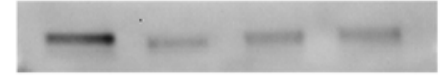

Bcl-2

GAPDH
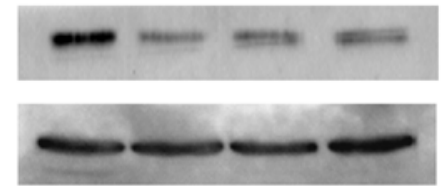

.
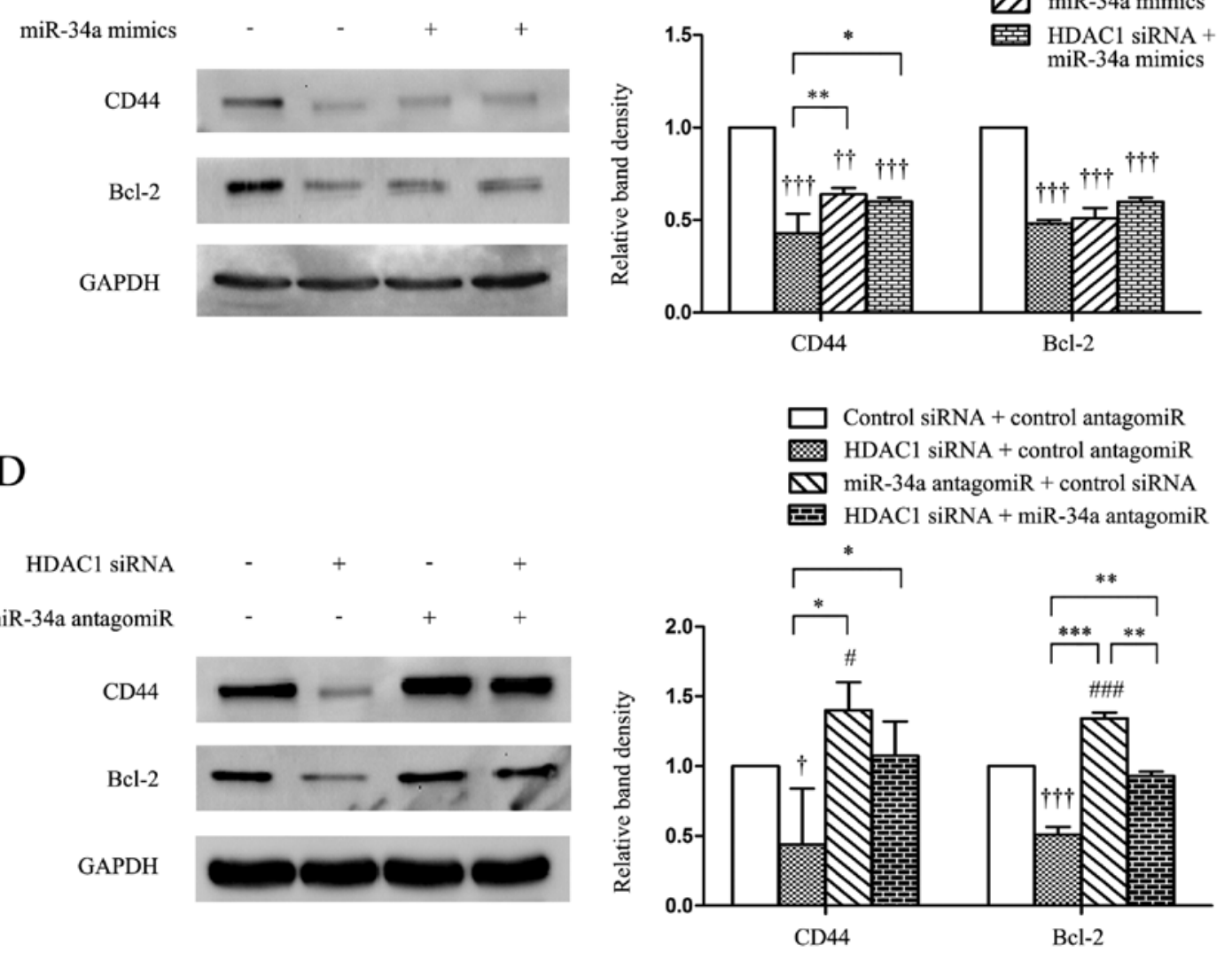

Figure 4. CD44 is regulated by miR-34a in gastric cancer cells. (A and B) SGC-7901 cells were transfected for $48 \mathrm{~h}$ with control or miR-34a mimics (A) or control, or miR-34a antagomiRs (B), and then subjected to immunoblotting. (C and D) Cells were transfected for $48 \mathrm{~h}$ with control or HDAC1 siRNAs, in the presence or absence of miR-34a mimics (C) or in the presence of control or miR-34a antagomiRs (D), and then subjected to immunoblotting. The density of each band was measured and normalized to GAPDH. The relative band density from control cells was defined as 1 . ${ }^{\dagger} \mathrm{P}<0.05$, ${ }^{\dagger \dagger} \mathrm{P}<0.01$ and ${ }^{\dagger \dagger} \mathrm{P}<0.001, \mathrm{a}$ significant reduction; and ${ }^{\#} \mathrm{P}<0.05$ and ${ }^{\# \# \# /} \mathrm{P}<0.001$, a significant increase versus the controls. ${ }^{*} \mathrm{P}<0.05,{ }^{* *} \mathrm{P}<0.01$ and ${ }^{* * * *} \mathrm{P}<0.001$ indicate a significant difference. HDAC1, histone deacetylase 1.

target protein of miR-34 (10). Co-transfection of miR-34a mimics and HDAC1 siRNA did not significantly alter the expression levels of CD44 and Bcl-2, compared with miR-34a mimics alone, whereas it slightly reduced the expression of CD44, and significantly reduced the expression of Bcl-2, compared with miR-34a antagomiR alone (Fig. 4C and D).

HDAC1 and CD44 regulates proteins responsible for the cellular cytoskeleton. Transfection of HDAC1 or CD44 siRNA downregulated the expression of RhoA and P-LIMK-1, and reduced RhoA activity as evidenced by a lower level of
GTP-bound RhoA. However, it had no effect on the expression of LIMK-1, compared with the controls (Fig. 5A-C). The simultaneous depletion of HDAC1 and CD44 resulted in further downregulation of RhoA and P-LIMK. However, the level of GTP-bound RhoA in the double knockdown cells was only slightly lower than cells depleted of HDAC1 or CD44 alone (Fig. 5A-C). The immunoblotting results showed that the level of supernatant MMP-2 protein was reduced by the depletion of HDAC1 or CD44, and the results were supported by gelatin zymography assays, which showed that depletion of HDAC1 or CD44 reduced the level of active MMP-2 (Fig. 5D). 
A

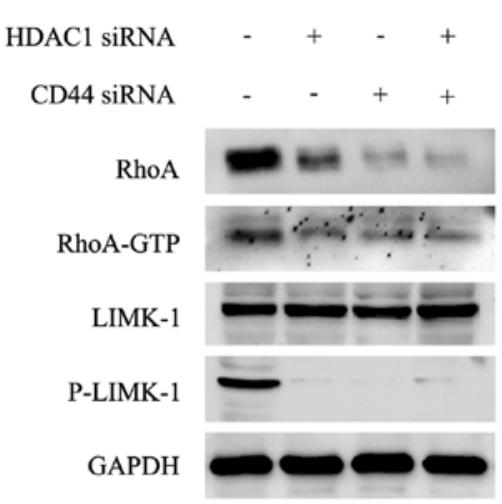

$\mathrm{C}$

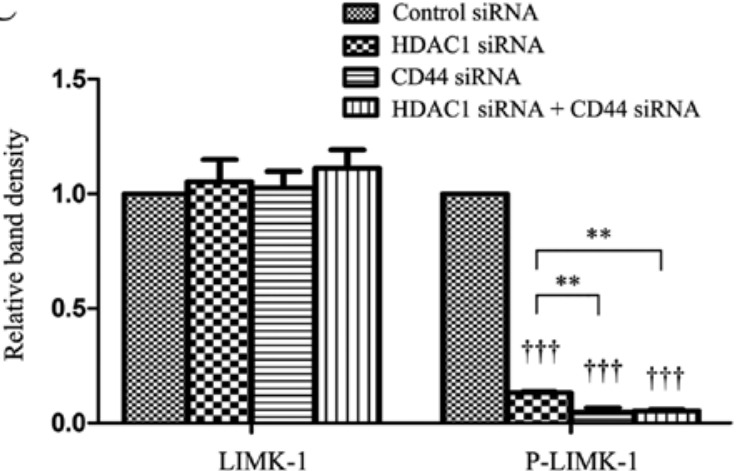

B
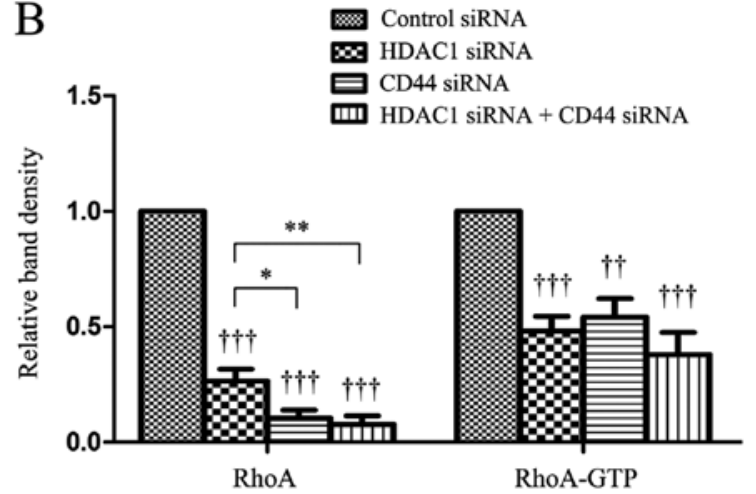

$\mathrm{D}$

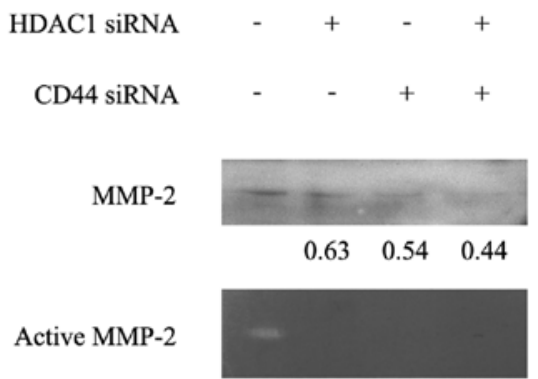

Figure 5. HDAC1 and CD44 regulate proteins responsible for the cellular cytoskeleton. (A) SGC-7901 cells were transfected for 48 h with control, HDAC1 or CD44 siRNAs, or HDAC1 siRNA + CD44 siRNA, and then subjected to immunoblotting. (B and C) The density of each band was measured and normalized to GAPDH. The relative band density from the control cells was defined as 1 . (D) The above cells were cultured in serum-free medium (starvation) for $24 \mathrm{~h}$ The conditioned medium was collected and subjected to gelatin zymography assays to detect active $\mathrm{MMP}-2$. ${ }^{\dagger} \mathrm{P}<0.01$ and ${ }^{\dagger} \mathrm{P}<0.001$ indicate a significant reduction versus the controls. ${ }^{*} \mathrm{P}<0.05$ and ${ }^{* *} \mathrm{P}<0.01$ indicate a significant difference. HDAC1, histone deacetylase 1 .

\section{Discussion}

The present study has, to the best of our knowledge, demonstrated for the first time that HDAC1 depletion inhibits the metastatic abilities of gastric cancer cells by regulating the miR-34a/CD44 pathway. Depletion of HDAC1 significantly upregulated the expression of miR-34a, and inhibited the abilities of gastric cancer cells to migrate, invade and adhere, impaired the organization of microfilaments and microtubules and impaired the formation of cellular pseudopodia. These effects were restored by a specific miR-34a antagomiR. The HDAC1 and miR-34a axis regulates the cellular cytoskeleton by mediating the expression and/or activation of CD44 and the downstream proteins including RhoA, LIMK and MMP-2.

Given that HDACs are overexpressed in cancer, inhibition of HDACs arrests cell cycling and often drives cancer but not normal cells, into cell death pathways, attention has been given to using HDAC inhibitors for cancer treatments (5). HDAC has also exhibited a profound impact on the expression of microRNAs, resulting in changed expression of $\sim 40 \%$ of these microRNAs (20). Pan-HDAC inhibitors have been demonstrated to regulate the expression of miRNAs in cells of colon carcinoma, lymphoma and breast cancer (21). In accordance, in the present study we have shown that depletion of HDAC1 by siRNA resulted in an altered expression of 101 differentially expressed miRNAs matching the 4-fold threshold in gastric cancer cells.
miR-34, a transcriptional target of $\mathrm{p} 53$, is a potent tumor suppressor that inhibits a broad range of cancer cells by repressing a plethora of oncogenes that control cell proliferation, senescence, apoptosis and metastasis $(10,22)$. The miR-34 family comprises three processed miRNAs that are encoded by two different genes: miR-34a is encoded by its own transcript, whereas miR-34b and miR-34c share a common primary transcript (10). miR-34a is often found inactivated in cancer cells (10). It has been reported that inhibition of HDAC1 mimics the miR-34a phenotype (22). In the present study, we have further demonstrated that depletion of HDAC1 upregulated the expression of miR-34a, but had little effect on the expression of miR-34b or miR34c, suggesting that overexpressed HDAC1 exerts its actions on gastric cancer cells by dysregulating miR-34a. The results are also supported by a study that Vorinostat, an HDAC inhibitor, restored the expression of miR-34a in pancreatic cancer stem cells (23).

The present study has also demonstrated that the antimetastatic effects of HDAC1 depletion are mediated by regulating the expression of CD44 via miR-34a. Consistently, the expression level of HDAC1 was positively correlated with that of CD44 in chronic lymphocytic leukemia (7) and HDAC1 inhibitors reduced the expression of CD44 via indirect mechanisms in hepatocellular carcinoma cells (24). CD44 is a direct and functional target of miR-34a as CD44 knockdown phenocopies miR-34a overexpression in inhibiting the metastasis of prostate cancer cells (25). 


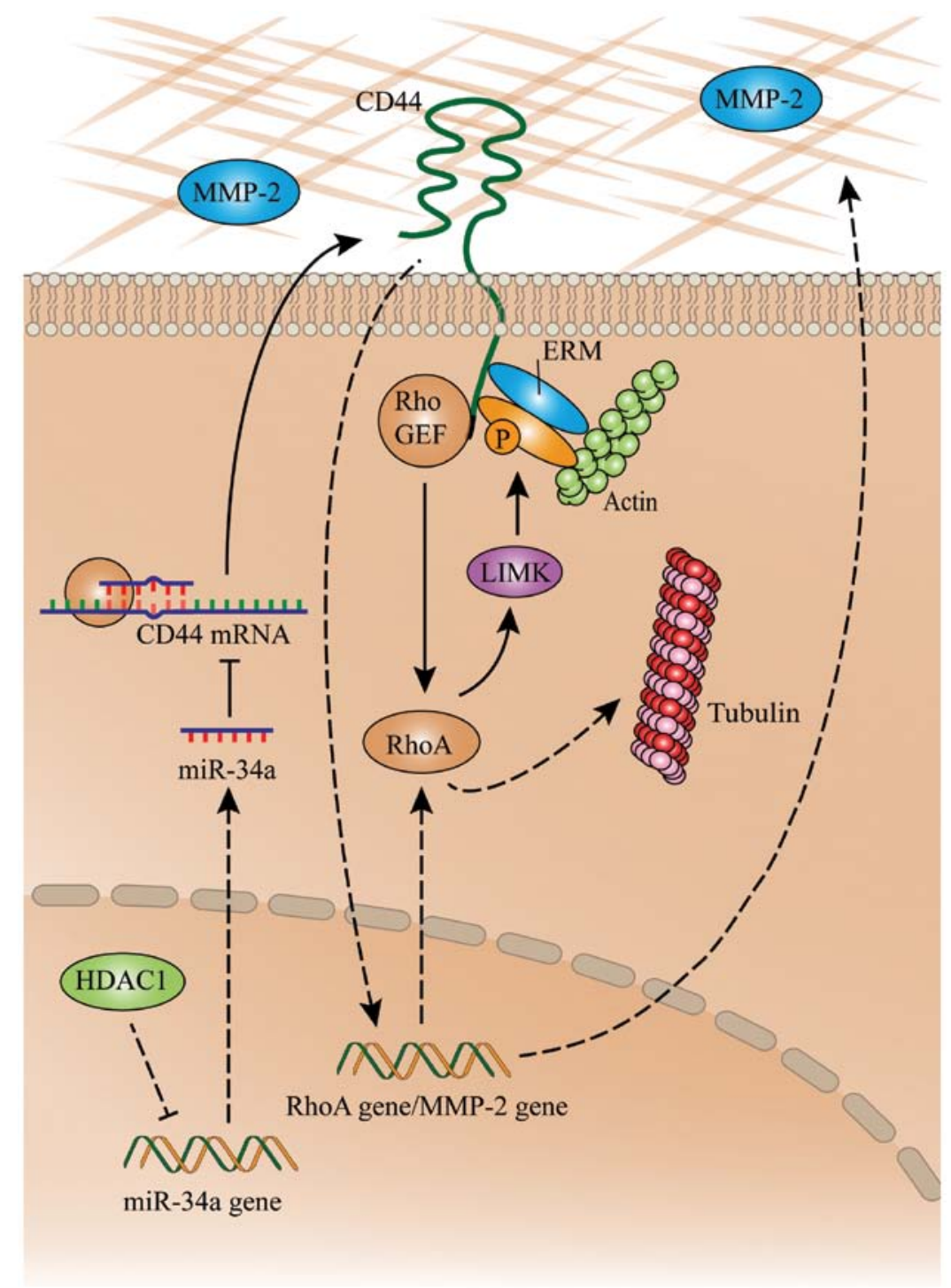

Figure 6. A schematic summary on the HDAC1/miR-34a/CD44 pathway in gastric cancer cells. ' $\rightarrow$ ' indicates positive regulation or activation; ' $\perp$ ', negative regulation or blockade. Solid lines indicate a direct regulation, while dashed lines, an indirect regulation. ERM, ezrin, radixin and moesin; HDAC1, histone deacetylase 1; LIMK, LIM domain kinase; miR, microRNA; MMP-2, matrix metalloproteinase-2; RhoA, ras homolog family member A.

CD44 is a cell-surface adhesion glycoprotein involved in cellular adhesion, migration and apoptosis resistance, and is highly expressed in numerous cancer cells (26). CD44 indirectly links to actin by interacting with ERM proteins (ezrin, radixin and moesin) (27). Knockdown of CD44 disturbs the organization of actin cytoskeleton and the formation of cellular pseudopodia (28). CD44 interacts with RhoGEF to activate RhoA, which modulates cell motility through the actin cytoskeleton, and promotes the lymphatic metastasis of human gastric cancer cells (29). The activated RhoA also activated LIMK-1, which is a key regulator of cytoskeletal organization involved in the migration and proliferation of cancer cells (30). In the present study, we have shown that depletion of CD44 also reduced the level of supernatant MMP-2 protein and the activity of MMP-2, thus attenuating the cell invasion ability since MMP-2 selectively increases the degradation of extracellular matrix components, and facilitates tumor invasion and metastasis (31).
In conclusion, the proposed mechanism by which depletion of HDAC1 inhibits metastatic abilities of gastric cancer cells via the miR-34a/CD44 pathway is shown in Fig. 6. HDAC1 downregulates the expression of miR-34a, which in turn dysregulates CD44 mRNA, leading to the overexpression of the CD44 protein. CD44 indirectly links to actin by interacting with ERM proteins (27), and activating RhoA by interacting with RhoGEF. Activated RhoA guides the polymerization of tubulins and actins by activating LIMK, which mediates the accumulation of microfilaments and microtubules and cellular pseudopodia $(32,33)$. CD44 also regulates the expression and activation of MMP-2 (34), which indirectly activates RhoA.

\section{Acknowledgements}

This study was supported by grants from the National Natural Scientific Foundation (81272467), and the Ministry of Health (201002015). 


\section{References}

1. Siegel R, Naishadham D and Jemal A: Cancer statistics, 2013. CA Cancer J Clin 63: 11-30, 2013.

2. Choudhary C, Kumar C, Gnad F, Nielsen ML, Rehman M, Walther TC, Olsen JV and Mann M: Lysine acetylation targets protein complexes and co-regulates major cellular functions. Science 325: 834-840, 2009.

3. Venugopal B and Evans TR: Developing histone deacetylase inhibitors as anti-cancer therapeutics. Curr Med Chem 18: $1658-1671,2011$.

4. Dovey OM, Foster CT and Cowley SM: Histone deacetylase 1 (HDAC1), but not HDAC2, controls embryonic stem cell differentiation. Proc Natl Acad Sci USA 107: 8242-8247, 2010

5. Delcuve GP, Khan DH and Davie JR: Targeting class I histone deacetylases in cancer therapy. Expert Opin Ther Targets 17: 29-41, 2013.

6. Weichert W, Röske A, Gekeler V, Beckers T, Ebert MP, Pross M, Dietel M, Denkert C and Röcken C: Association of patterns of class I histone deacetylase expression with patient prognosis in gastric cancer: A retrospective analysis. Lancet Oncol 9: 139-148, 2008.

7. Wang JC, Kafeel MI, Avezbakiyev B, Chen C, Sun Y, Rathnasabapathy C, Kalavar M, He Z, Burton J and Lichter S: Histone deacetylase in chronic lymphocytic leukemia. Oncology 81: 325-329, 2011.

8. Li T, Lu YY, Zhao XD, Guo HQ, Liu CH, Li H, Zhou L, Han YN, Wu KC, Nie YZ, et al: MicroRNA-296-5p increases proliferation in gastric cancer through repression of Caudal-related homeobox 1. Oncogene 33: 783-793, 2014.

9. Garzon R, Marcucci G and Croce CM: Targeting microRNAs in cancer: Rationale, strategies and challenges. Nat Rev Drug Discov 9: 775-789, 2010.

10. Hermeking H: The miR-34 family in cancer and apoptosis. Cell Death Differ 17: 193-199, 2010.

11. Buurman R, Gürlevik E, Schäffer V, Eilers M, Sandbothe M, Kreipe H, Wilkens L, Schlegelberger B, Kühnel F and Skawran B: Histone deacetylases activate hepatocyte growth factor signaling by repressing microRNA-449 in hepatocellular carcinoma cells Gastroenterology 143: 811-20.e1, 15, 2012.

12. Wang Y, Toh HC, Chow P, Chung AY, Meyers DJ, Cole PA, Ooi LL and Lee CG: MicroRNA-224 is up-regulated in hepatocellular carcinoma through epigenetic mechanisms. FASEB J 26 3032-3041, 2012

13. Mims A, Walker AR, Huang X, Sun J, Wang H, Santhanam R, Dorrance AM, Walker C, Hoellerbauer P, Tarighat SS, et al: Increased anti-leukemic activity of decitabine via AR-42-induced upregulation of miR-29b: A novel epigenetic-targeting approach in acute myeloid leukemia. Leukemia 27: 871-878, 2013.

14. Chakraborty S, Mazumdar M, Mukherjee S, Bhattacharjee $P$, Adhikary A, Manna A, Chakraborty S, Khan P, Sen A and Das T: Restoration of $\mathrm{p53} / \mathrm{miR}-34 \mathrm{a}$ regulatory axis decreases survival advantage and ensures Bax-dependent apoptosis of non-small cell lung carcinoma cells. FEBS Lett 588: 549-559, 2014.

15. LeBoeuf M, Terrell A, Trivedi S, Sinha S, Epstein JA, Olson EN, Morrisey EE and Millar SE: Hdac1 and Hdac2 act redundantly to control p63 and p53 functions in epidermal progenitor cells. Dev Cell 19: 807-818, 2010.

16. Zhai B, Hu F, Jiang X, Xu J, Zhao D, Liu B, Pan S, Dong X, Tan $\mathrm{G}$, Wei Z, et al: Inhibition of Akt reverses the acquired resistance to sorafenib by switching protective autophagy to autophagic cell death in hepatocellular carcinoma. Mol Cancer Ther 13: 1589-1598, 2014.
17. Zhao D, Zhai B, He C, Tan G, Jiang X, Pan S, Dong X, Wei Z, Ma L, Qiao H, et al: Upregulation of HIF-2 $\alpha$ induced by sorafenib contributes to the resistance by activating the TGF- $\alpha /$ EGFR pathway in hepatocellular carcinoma cells. Cell Signal 26: 1030-1039, 2014

18. Wei Z, Jiang X, Liu F, Qiao H, Zhou B, Zhai B, Zhang L, Zhang X, Han L, Jiang H, et al: Downregulation of Skp2 inhibits the growth and metastasis of gastric cancer cells in vitro and in vivo. Tumour Biol 34: 181-192, 2013.

19. Naor D, Nedvetzki S, Golan I, Melnik L and Faitelson Y: CD44 in cancer. Crit Rev Clin Lab Sci 39: 527-579, 2002.

20. Scott GK, Mattie MD, Berger CE, Benz SC and Benz CC: Rapid alteration of microRNA levels by histone deacetylase inhibition. Cancer Res 66: 1277-1281, 2006.

21. Izzotti A, Cartiglia C, Steele VE and De Flora S: MicroRNAs as targets for dietary and pharmacological inhibitors of mutagenesis and carcinogenesis. Mutat Res 751: 287-303, 2012.

22. Zhao J,Lammers P,Torrance CJ and Bader AG: TP53-independent function of miR-34a via HDAC1 and $\mathrm{p} 21^{\mathrm{CIP1} / \mathrm{WAF} 1}$. Mol Ther 21: 1678-1686, 2013

23. Nalls D, Tang SN, Rodova M, Srivastava RK and Shankar S: Targeting epigenetic regulation of miR-34a for treatment of pancreatic cancer by inhibition of pancreatic cancer stem cells. PLoS One 6: e24099, 2011.

24. Zeng SS, Yamashita T, Kondo M, Nio K, Hayashi T, Hara Y, Nomura Y, Yoshida M, Hayashi T, Oishi N, et al: The transcription factor SALL4 regulates stemness of EpCAM-positive hepatocellular carcinoma. J Hepatol 60: 127-134, 2014.

25. Liu C, Kelnar K, Liu B, Chen X, Calhoun-Davis T, Li H, Patrawala L, Yan H, Jeter C, Honorio S, et al: The microRNA miR-34a inhibits prostate cancer stem cells and metastasis by directly repressing CD44. Nat Med 17: 211-215, 2011.

26. Zhang Y, Xia H, Ge X, Chen Q, Yuan D, Chen Q, Leng W, Chen L, Tang Q and Bi F: CD44 acts through RhoA to regulate YAP signaling. Cell Signal 26: 2504-2513, 2014.

27. Zöller M: CD44: Can a cancer-initiating cell profit from an abundantly expressed molecule? Nat Rev Cancer 11: 254-267, 2011.

28. Sumoza-Toledo A and Santos-Argumedo L: The spreading of B lymphocytes induced by CD44 cross-linking requires actin, tubulin, and vimentin rearrangements. J Leukoc Biol 75: 233-239, 2004.

29. Lin MT, Lin BR, Chang CC, Chu CY, Su HJ, Chen ST, Jeng YM and Kuo ML: IL-6 induces AGS gastric cancer cell invasion via activation of the $\mathrm{c}-\mathrm{Src} / \mathrm{RhoA} / \mathrm{ROCK}$ signaling pathway. Int J Cancer 120: 2600-2608, 2007.

30. McConnell BV, Koto K and Gutierrez-Hartmann A: Nuclear and cytoplasmic LIMK1 enhances human breast cancer progression. Mol Cancer 10: 75, 2011.

31. Murphy $\mathrm{G}$ and Nagase $\mathrm{H}$ : Progress in matrix metalloproteinase research. Mol Aspects Med 29: 290-308, 2008.

32. Hanna S and El-Sibai M: Signaling networks of Rho GTPases in cell motility. Cell Signal 25: 1955-1961, 2013.

33. Struckhoff AP, Rana MK and Worthylake RA: RhoA can lead the way in tumor cell invasion and metastasis. Front Biosci 16: 1915-1926, 2011.

34. Kim Y, Lee YS, Choe J, Lee H, Kim YM and Jeoung D: $\mathrm{CD} 44$-epidermal growth factor receptor interaction mediates hyaluronic acid-promoted cell motility by activating protein kinase $\mathrm{C}$ signaling involving Akt, Racl, Phox, reactive oxygen species, focal adhesion kinase, and MMP-2. J Biol Chem 283: 22513-22528, 2008 